\title{
Numerical and experimental studies on a Syngas-fired Ultra low $\mathrm{NO}_{\mathrm{x}}$ combustor
}

\author{
Krishna Sesha Giri \\ Post-Doctoral Research Fellow \\ Clean Combustion Research Center, \\ King Abdullah University of Science and Technology, \\ Saudi Arabia \\ krishna.seshagiri@kaust.edu.sa
}

\author{
R V Ravikrishna \\ Professor \\ Department of Mechanical Engineering \\ Indian Institute of Science, Bangalore, India \\ ravikris@mecheng.iisc.ernet.in
}

\begin{abstract}
Simulations and exhaust measurements of temperature and pollutants in a syngas-fired model trapped vortex combustor for stationary power generation applications are reported. Numerical simulations employing RANS and LES with Presumed Probability Distribution Function (PPDF) model were also carried out. Mixture fraction profiles in the TVC cavity for non-reacting conditions show that LES simulations are able to capture the mean mixing field better than the RANS-based approach. This is attributed to the prediction of the jet decay rate and is reflected on the mean velocity magnitude fields, which reinforce this observation at different sections in the cavity. Both RANS and LES simulations show close agreement with the experimentally measured $\mathrm{OH}$ concentration, however, the RANS approach does not perform satisfactorily in capturing the trend of velocity magnitude. LES simulations satisfactorily capture the trend observed in exhaust measurements which is primarily attributed to the flame stabilization mechanism. In the exhaust measurements, mixing enhancement struts were employed and their effect evaluated. The exhaust temperature pattern factor was found to be poor for baseline cases, but improved with the introduction of struts. NO emissions were steadily below 3-ppm across various flow conditions, whereas $\mathrm{CO}$ emissions tended to increase with increasing Momentum Flux Ratios (MFRs) and mainstream fuel addition. Combustion efficiencies 96\% were observed for all conditions. The performance characteristics were found to be favourable at higher MFRs with low pattern factors and high combustion efficiencies.

Keywords: Trapped vortex combustor, Syngas, Presumed Probability Density Function (PPDF), Low NOx combustor, Reynolds averaged Navier Stokes (RANS); Large Eddy Simulations (LES)
\end{abstract}




\section{Introduction}

Renewable energy-based stationary power generation systems are very important in the current scenario of fossil fuel crisis. The development of high pressure gasification systems has led to efficient methods of obtaining gaseous syngas fuel from various biomass feedstocks. Integrated gasification combined cycle (IGCC) combines the advantage of a high pressure gasification system with gas turbine engine cycle for stationary power generation applications. The waste heat from the gasifier and the gas turbine cycle is utilized in a recovery steam turbine cycle. This concept is aimed at maximum utilization of energy and the efficiencies of the combined cycle are found to be high $(\sim 45 \%)$.

It is essential to ensure complete and stable combustion of syngas fuel in the gas turbine engine combustor to ensure low pollutant emissions with high combustion efficiencies. The Trapped Vortex Combustor (TVC) is a gas turbine combustor concept which provides very good flame stability at wide equivalence ratio conditions. Earlier works have reported that the TVC concept aids in obtaining low pollutant emissions, with ultra-low NOx particularly [1-5]. One of our previous works reported the cavity structures measured using acetone PLIF and PIV techniques in a single cavity TVC by defining a parameter namely the momentum flux ratio (MFR) [6]. A clear trend based on the MFR was observed and the same has been established with sufficient rigor. Subsequently we carried out OH-PLIF studies and reported flame stabilization regimes in the cavity based on $\mathrm{OH}$ concentration fields [7].

Jin et al. [13] reported PIV and exhaust measurement studies on a liquid-fuelled model trapped vortex combustor. They employed radial struts to enhance mixing and provide favourable pattern factors at the exit which they attributed to the location of dilution holes. Nemitallah et al. [14] reported experimental and numerical studies of oxy-fuel combustion in a model gas turbine combustor. The flame stabilization in the combustor were reported based on the oxygen percentage and was found to extinguish for composition of < $21 \%$. Numerical simulations with modified two-step models satisfactorily captured the temperature and overall flame shape. Zeinivand et al. [15] evaluated the effect of stabilizer jets on NOx emissions in a jet-stabilized combustor using a Finite Volume staggered grid approach. It was found that as the axial distance of the stabilizer jets from the fuel injector increases, NOx emissions tend to decrease. Zhang et al. [16] reported the development of a dual vortex combustor using a pilot combustion zone. They showed that the preheat temperature and staging of air influences the LBO limits and other performance characteristics. Arghode et al. [17] reported colourless distributed combustion in a high intensity combustor. They were able to obtain high thermal intensity while ensuring low emissions of $<8-\mathrm{ppm} \mathrm{NO}_{\mathrm{x}}$ at desirable conditions. To summarize, there is a dearth of literature on simulations reporting mixing fields under non-reacting conditions and species concentration under reacting conditions in trapped vortex combustors. More importantly, there is no previous study using syngas in TVC, and hence, this work represents the first such study to the best of our knowledge. The present work not only reports experimental data, but combines it with detailed modelling using both RANS and LES approaches. This is considered important in the present context since there is a need to explain combustor performance based on cavity and mixing dynamics as observed from simulations. 
The present work reports exhaust measurements of temperature and pollutants in a single cavity trapped vortex combustor employing syngas fuel to evaluate its performance. This is the first work to present performance characteristics of such a combustor and comparison with numerical simulations. The relevance to novel power generation concepts such as IGCC is the most important aspect of this article. We present exhaust measurements of temperature and pollutants in a single cavity trapped vortex combustor to evaluate its performance. Specifically, the exhaust pattern factor and average CO, THC and NO levels are reported and performance enhancement obtained using struts which improve mixing in the combustor is also shown. The current work also employs LES with Presumed Probability Distribution Function (PPDF) models to model the turbulent combustion inside the combustor. After reporting the combustor performance, the paper describes results of numerical simulations and the comparison of predictions with previously reported measurements of mixture fraction, $\mathrm{OH}$ concentrations and velocity under non-reacting and reacting conditions. The paper concludes by explaining the basis for the low $\mathrm{NO}_{\mathrm{x}}$ emission characteristics and favourable exhaust pattern factor using observations from simulations.

\section{Numerical studies: RANS and LES simulations}

This section discusses the results of numerical simulations carried out using RANS and LES framework and the comparison of these results with previous experiments [6, 7]. Insights are also obtained from the simulations to explain the results of the exhaust measurements reported in the next section. The simulations are carried out using the Star CCM+ CFD code. In the case of RANS simulations, Realizable k- $\epsilon$ turbulence model and Large Eddy Simulations with WALE sub-grid scale model have been used with PPDF based flamelet library model for reacting flow cases. The Realizable $k-\epsilon$ turbulence model is known to predict jet decay in the case of round jets satisfactorily. The WALE subgrid scale model is the preferred model in channel flow geometries and hence the same has been employed as the sub-grid scale model. The adiabatic PPDF flamelet libraries are generated using DARS-CFD package for different scalar dissipation rates based on a presumed probability distribution. In the mixture fraction space, the species mass fraction conservation equation is given by:

$$
\frac{\partial Y_{i}}{\partial t}=R_{i, k i n}+D_{i} \chi \frac{\partial^{2} Y_{i}}{\partial f^{2}},
$$

where $\mathrm{Y}_{\mathrm{i}}$ is the species mass fraction of the $i^{\text {th }}$ species, $\mathrm{R}$ is the net rate of production or consumption of the species, $\mathrm{D}$ is the mass diffusivity, $\chi$ is the scalar dissipation rate, and $f$ is the mixture fraction.

The energy conservation equation is given by:

$$
\frac{\partial T}{\partial t}=\left[-\sum_{i=1}^{N} \frac{h_{i} R_{i, k i n}}{C_{p}}\right]+\alpha \chi\left(\frac{\partial^{2} T}{\partial f^{2}}\right)
$$

where $T$ is the cell temperature, $h$ is the specific enthalpy of the species, and $C_{p}$ is the specific heat capacity, and $\alpha$ is the thermal conductivity. The scalar dissipation rate is given by:

$$
\chi=(\nabla f . \nabla f)
$$


The flamelet libraries are generated for different scalar dissipation rates along the mixture fraction space so that any quantity $\varphi=\varphi(f, \chi)$, a function of the mixture fraction and scalar dissipation rate. The chemical kinetic mechanism employed in this work is due to Li et al. [8] with 12 species and 33 reactions. The effect of kinetic mechanism on the temperature predictions is very minimal as observed by Cuoci et al. [9]. However, species predictions tend to be affected by kinetic mechanisms. The mechanism employed here does not account for NOx production. It should also be noted that the STAR-CCM+ simulations were steady state simulations and were used as initial conditions for the LES simulations. Validation of the laminar flamelet model was carried out on the Sandia/ETH Zurich flame, a turbulent non-premixed, non-piloted $\mathrm{CO} / \mathrm{H}_{2} / \mathrm{N}_{2}$ flame in a previous work [18]. The validation simulations indicate that the flamelet model is suitable for predictions of temperature and major species.

\subsection{Boundary conditions, Computational domain and Grid Independence study}

The inlet temperature is $300 \mathrm{~K}$ for both fuel and air and the combustor pressure is atmospheric. The velocity boundary conditions are provided in Table 1 . The fuel composition by volume is CO-20\%, $\mathrm{H}_{2}-20 \%, \mathrm{CO}_{2}-12 \%$, $\mathrm{CH}_{4}-2 \%$ and the rest $\mathrm{N}_{2}$. The walls are treated as adiabatic with a no slip boundary. The experiments were conducted for a short duration ( $\sim 1$ minute) and hence, the heat transfer to the wall is negligible during this brief period. The exit of the combustor has a pressure outflow boundary condition with ambient atmospheric pressure. The fluid properties are calculated by mass averaging. The chemical kinetic mechanism is provided as a CHEMKIN input file, and the same is used for generating flamelet libraries using DARS-CFD. The STAR$\mathrm{CCM}+$ solver employs a $2^{\text {nd }}$ order spatial discretization. The time stepping is implicit. The convective and diffusive flux are calculated using a bounded central difference scheme (BCD) in the case of LES simulations and using a $2^{\text {nd }}$ order upwind scheme in the case of RANS simulations.

A schematic of the model single cavity TVC is shown in Fig 1. It consists of a rectangular duct of cross-section 120-mm x 50-mm for the mainstream flow. A cavity of depth 70-mm and L/D of 1 is located at the bottom of the main flow duct. The cavity dimensions are chosen to represent a cross section of a compact combustor with application in micro gas turbines. The cavity fuel injection is through 6 holes of 10-mm diameter which are placed $15-\mathrm{mm}$ from the bottom of the cavity on the upstream wall. The guide vane which directs a portion of the flow into the cavity has a flow cross section of $10-\mathrm{mm} \times 120-\mathrm{mm}$. The configuration and placement of the guide vane has been optimized in the investigations by Agarwal and Ravikrishna [4, 5] and the same arrangement is used in the present study.

The grid generation was achieved using the inbuilt preprocessor in STAR-CCM+. The wall $\mathrm{y}+$ was set at $0.05-$ $\mathrm{mm}$ with a minimum cell size of $0.1-\mathrm{mm}$. The cell size expansion rate, which is the ratio of the cell characteristic dimension from one prism layer to the subsequent one proceeding from the wall, was maintained at 0.5. Grid independence study was carried out for CCM-RANS and LES simulations. The grid independence study was carried out with the following number of cells for different grid resolutions

1. Coarse grid -1.2 million cells

2. Intermediate grid -2 million cells

3. Fine grid -4 million cells

GTP-16-1015

Sesha Giri 
The near wall grid resolution was kept fine enough for boundary layer treatment. The computational cells are hexahedral elements inside the domain and polyhedral at the fuel inlet jets. In LES simulations, the grid size corresponds to the filter size and hence length scales smaller than the grid size locally are modelled using a subgrid scale model (WALE in this case). The typical grid size in the case of CCM simulations is $\sim 1 \mathrm{~mm}$ with finer grid sizes near the fuel inlet and walls. The results of the grid independence study are presented in Fig. 2 . The grid independence study shows that the intermediate and fine grids in both cases have approached convergence. Hence, the intermediate grid resolution was employed for further simulations. It has been shown in previous studies on LES reacting flow simulations in free shear [11] and complex combustor geometry [12] that the mean fields of temperature, species and velocity approach an asymptotic convergence with finer grid resolutions. This can be observed in the grid independence results of CCM-LES simulations where the intermediate and fine grid profiles show close agreement.

\subsection{Results and Discussion}

\section{Non-reacting flow simulations: Mixture fraction profile}

The non-reacting flow simulations using Realizable k- $\varepsilon$ model and Large Eddy simulations (WALE sub-grid scale model) were carried out with the intermediate grid resolutions mentioned in the previous section for the conditions mentioned in Table 1.

Simulation conditions have been chosen to include high to low MFRs corresponding to experimental measurements $[6,7]$ of mixture fraction, velocity field under non-reacting conditions and $\mathrm{OH}$ concentration and velocity field under reacting conditions. The results of simulations are presented in the form of profiles along the sections shown in Fig. 3. The three sections along the depth of the cavity correspond to the fuel inlet, exit of guide vane and cavity exit. In the span-wise direction, the section passes through the fuel inlet located at 51-mm from the front of the cavity corresponding to the plane of measurement of mixture fraction, velocity field and $\mathrm{OH}$ concentration. In Fig. 4 and subsequent figures, the simulations are compared with experimental data reported earlier $[6,7]$. Specifically, this data includes mean mixture fraction distribution obtained using Acetone Planar Laser Induced Fluorescence (PLIF), velocity data using Particle Image Velocimetry (PIV) and OH PLIF concentration contours in the cavity.

Figure 4 shows the snapshots of steady state CO mass fraction contours and velocity magnitude for Case 1 corresponding to MFR 4.5. It must be noted that in the case of LES simulations mean fields with sufficient temporal averaging have been reported for comparison with other simulations and experiments. It can be seen that RANS simulations differ slightly from LES simulations based on the contours of CO mass fraction. The fuel jet deviates to a large extent towards the lower wall of the cavity in the case of RANS. This observation can be extended to the velocity magnitude contours which also show small differences in the case of RANS. The deflection of the cavity jet due to the cross flow from the guide vane is a function of the MFR. At higher MFRs the jet deflection is negligible as seen from LES simulations. However, this is slightly over-predicted by the RANS simulations. The velocity magnitude contours show higher velocities for the cross flow stream in the case of RANS simulations which results in the erroneous prediction of jet deflection. It is essential to 
compare the profiles of mixture fraction at various locations to identify the simulation approach that provides closest agreement. The mixture fraction given by the following equation denotes the extent of mixing of fuel and air.

$$
Z=\left[\frac{s Y_{F}-Y_{O}+Y_{O, 0}}{s Y_{F, 0}+Y_{O, 0}}\right]
$$

where $\mathrm{Z}$ is the mixture fraction, $\mathrm{Y}_{\mathrm{f}}$ is the fuel mass fraction, $\mathrm{Y}_{\mathrm{o}}$ is the oxidizer mass fraction, $\mathrm{Y}_{\mathrm{f}, 0}$ is the fuel mass fraction at fuel inlet and $\mathrm{Y}_{\mathrm{o}, 0}$ is the oxidizer mass fraction at the air inlet and $\mathrm{s}$ is the stoichiometric airfuel ratio. The simulation results of mixture fractions are compared with experimental measurements in the subsequent section

Figure 5 shows the profiles of mixture fraction at two sections for MFRs 4.5, 1.1 and 0.3. The top panel shows the contours for MFR=4.5 at section 1 and 2 shown in Fig. 4. As observed in Fig. 4, the fuel jet bending is over-predicted which is evident from the dip in the mixture fraction profile for RANS simulations. However, this is not seen in the case of experimental measurements. The mixture fraction profile from experiments is matched closest by the LES simulations. This is attributed to the effect of turbulent mixing at finer scales which is resolved in the case of LES simulations whereas the same is modeled in RANS approach. The turbulent mixing process contributes to the mixing phenomenon to a larger extent as compared to mixing due to diffusion which accounts for the slight difference in the mixture fraction values between LES simulations and experiments. It must be noted that a Lewis number of 1 is assumed in all simulations. The jet deflection increases with decreasing MFRs (not shown here). This trend can be seen in the mixture fraction contours as well in Fig. 5.

The middle panel corresponds to the profiles of mixture fraction for Case 2 corresponding to an MFR of 1.1. It can be seen that the onset of the fuel jet deflection is shifted towards an upstream location when compared to the MFR=4.5 case. This is captured satisfactorily by CCM simulations using both RANS and LES approach. This observation can be made in the case of Section 3 as well which shows the mixture fraction profiles at the cavity exit plane. The bottom panel in Fig. 5 shows mixture fraction profiles for MFR 0.3 at sections 1 and 2. A similar trend is seen in the low MFR case as well. It can be concluded based on Fig. 5 that LES simulations tend to agree well with experimental mixing contours followed by simulations using Realizable k- $\varepsilon$ model Figure 6 shows the typical velocity magnitude profiles at Section 2 for the three MFR cases. It would be worthwhile to study Fig. 6 in conjunction with Fig. 5 by studying the mixture fraction profiles as a result of the velocity field. For instance in the case of $M F R=4.5$, the LES simulation shows close agreement with the experimental values whereas the RANS simulations with Realizable k- $\varepsilon$ model differ from the measurements. This deviation in the velocity field prediction in turn affects the predictions of the mixing fields as seen earlier. In case of MFRs of 1.1 and 0.3 as well, the velocity magnitudes are predicted well by LES simulations as compared to RANS simulations, especially in Case 3 which corresponds to a low MFR of 0.3. 
The non-reacting flow simulations show that the LES predictions match well with the experiments except at the near wall regions where the experimental uncertainties are high. The qualitative agreement is within $15 \%$ in all other regions of the cavity.

\section{Reacting flow simulations: $\mathrm{OH}$ concentration profiles}

The reacting flow simulations with PPDF model were carried out and the results are presented in the form of $\mathrm{OH}$ concentration profiles together with velocity magnitude profiles. The chemical kinetic mechanism is due to Li et al. [8]. The PPDF flamelet libraries are generated for different scalar dissipation rates in the mixture fraction space using DARS-CFD package and imported to Star-CCM+. Figure 7 shows the $\mathrm{OH}$ concentration contours for Case 1 corresponding to a MFR of 4.5.

It can be seen that simulations and experiments show similar $\mathrm{OH}$ contours in terms of location of the peak $\mathrm{OH}$ concentration. Previous studies [6,7] have pointed out that a non-premixed diffusion controlled flame is stabilized in the high MFR cases based on the flow field. The fuel and the air streams are separated by the flame front at the interface. A flame front is also observed at the trailing edge of the fuel jet due to the stabilizing effect of the tail wake region. The PPDF model predictions in STAR-CCM+ simulations depend on the mixture fraction field which is in turn dependent on the flow field predictions. As observed in the previous section the Realizable k- $\varepsilon$ model and LES-WALE sub-grid scale model predict flow field with close agreement to experimental measurements and hence, $\mathrm{OH}$ concentration fields are also predicted precisely. However, the effect of the high temperature due to the reactions alters the flow field and hence the mixture fractions fields as well.

Figure 8 shows the $\mathrm{OH}$ concentration contours for Case 2 corresponding to an MFR of 1.1. The experimental OH PLIF contours show a high concentration at the downstream region of the cavity. This is predicted well by both RANS and LES simulations. This trend is clear from $\mathrm{OH}$ concentration profiles shown in Figure 9.

Figure 9 shows the profiles of $\mathrm{OH}$ concentration at Sections 2 and 3 for MFRs 4.5, 1.1 and 0.3. The top panel corresponds to MFR of 4.5. The OH concentration profile at Section 2 shows that both RANS and LES simulations agree pretty well with the experimental measurements. The CCM-RANS simulations capture the location of the reaction zone and the thickness in addition to the peak value. The CCM-LES simulations also agree with the location and peak value of the $\mathrm{OH}$ concentration. However, the flame is reduced in thickness considerably. This is a result of the LES model resolving length scales larger than the grid size while the RANS simulations model all scales. The finer mixing scales are captured and hence, the reaction zone is substantially reduced. At Section 3, as well the OH profiles match well with both CCM-RANS and LES simulations.

The middle panel shows the $\mathrm{OH}$ profiles for MFR=1.1. It can be seen that RANS simulations slightly underpredict the $\mathrm{OH}$ concentration values at both sections. At a MFR of 1.1, the reaction zone is stabilized where the fuel and air is considerably premixed before combustion. Hence, a fuel-air partially premixed charge undergoes reaction at the downstream portion of the cavity. Both RANS and LES simulations capture the flame location and peak values considerably well. In the case of LES simulations, the mean $\mathrm{OH}$ concentration fields give rise to multiple peaks of $\mathrm{OH}$ concentration. However, it must be noted that the high $\mathrm{OH}$ concentration regions lies within the peak $\mathrm{OH}$ region from experiments and a large enough temporal averaging tends to smear GTP-16-1015 Sesha Giri 
out the multiple peaks. The bottom panel shows a similar trend with a low MFR of 0.3 and enhanced fuel-air premixing. The RANS and LES simulations provide good predictions at both sections 2 and 3.

These observations are reinforced by velocity magnitude profiles for the reacting flow in Fig. 10. The velocity magnitude profiles show that both RANS and LES simulations do not show a close enough match with experimental measurements. The effect of heat release due to the chemical reactions is seen in the flow field predictions.

The preceding discussion outlines the effect of MFR on the mixture fraction, velocity magnitude and OH concentration profiles. The LES simulations show good agreement with the experimental measurements in terms of the qualitative trends. However, the quantitative values are different in some cases ( $30 \%$ for the peak values for reacting flow cases, and $15 \%$ for non-reacting flow cases) which has been discussed in the previous section

Based on the exhaust measurements and simulations, it is clear that at higher MFRs, the mixing is relatively low, and hence, the exhaust CO emissions are high. It is imperative that the combustor must be operated at lower MFRs to obtain the advantage of lower CO levels due to better mixing, and better exhaust patter factor as well. The NO levels do not vary with the MFRs due to the RQL strategy. From the practical operation standpoint, the combustor must be longer to ensure complete combustion.

\section{Experimental studies: Combustor performance evaluation}

A description of the combustor geometry is provided in the previous section and shown in Fig 1. In this section the combustor is evaluated in terms of pollutant emissions and exhaust temperature pattern factor. The measurements are carried out at the exit section of the combustor. Figure 11(a) shows locations of thermocouples at the exit cross section. The temperature measurements correspond to an average of the two thermocouple readings in the span-wise direction as shown in Fig. 11. Four uncoated B-type thermocouples of wire size $0.25 \mathrm{~mm}$ have been used. A butt-weld junction is used which provides faster response compared to a bead-type junction. B-type thermocouples can be used to measure temperatures in the range of $0-1700^{\circ} \mathrm{C}$ continuously and up to $1800^{\circ} \mathrm{C}$ for short duration. The two dissimilar metals forming the thermocouple junction are platinum $+30 \%$ rhodium vs. platinum $+6 \%$ rhodium. A National Instruments (NI) data acquisition system (TB 4353) is used along with thermocouples to acquire temperature data with built-in cold junction compensation. The mean of 100 samples at an acquisition rate of $17 \mathrm{~Hz}$ is reported here. The radiation correction for the temperature reading is done using the following relation

$$
T_{g}=T_{t}+\frac{e \sigma D}{\lambda N u}\left[T_{t}^{4}-T_{a}^{4}\right]
$$

where $T_{g}$ is the actual gas temperature, $T_{t}$ is measured, $T_{a}$ is the temperature of the surroundings, e is a constant ( 0.2 for non-coated thermocouple bead), $\sigma$ is the Stefan-Boltzmann constant $\left(5.67 \times 10 \mathrm{E}-8 \mathrm{~W} / \mathrm{m}^{2}-\mathrm{K}^{4}\right), \lambda$ is gas thermal conductivity, $\mathrm{Nu}$ is the local Nusselt number, and D is the thermocouple bead diameter.

For measurement of exhaust emission from the TVC rig, an emission analyser (Analyzer Instruments Company Ltd. Kota, India) is used. This analyser consists of different gas analyser modules with a heated line and 
diaphragm pump for sampling. The concentrations of $\mathrm{CO}$ (Non dispersive Infrared sensor - 0-3000 ppm) $\mathrm{CO}_{2}$ (Non dispersive Infrared sensor - 0-20\%), O2 (Paramagnetic sensor- 0-25\%), NO (Chemiluminescence method- 0-100 ppm), THC (Flame ionisation detector - 0-1000 ppm) are measured and logged using a data acquisition system (Ambitronics Ltd) using a RS-232 interface. Electrical heating ensures that wet readings are taken (which is the adopted convention in the present work). The acquisition of data is carried out after all values attain steady state. The exhaust pattern factor is defined as follows:

$$
\text { Pattern Factor (P.F) }=\frac{T_{m a x}-T_{a v}}{T_{a v}-T_{i n}}
$$

where $\mathrm{T}_{\max }$ is the maximum temperature, $\mathrm{T}_{\mathrm{av}}$ is the average temperature and $\mathrm{T}_{\mathrm{in}}$ is the inlet temperature for the combustor. Exhaust measurements were carried out for various cases depending on momentum flux ratio (MFR) which is defined as follows:

$$
\text { Momentum flux ratio }(M F R)=\frac{\rho_{f} v_{c f}^{2}}{\rho_{g v} v_{g v}^{2}}
$$

where $\mathrm{v}_{\mathrm{cf}}$ is the cavity fuel jet velocity $(\mathrm{m} / \mathrm{s}), \mathrm{v}_{\mathrm{gv}}$ is the guide vane air stream velocity $(\mathrm{m} / \mathrm{s}), \rho_{\mathrm{f}}$ is the density of the fuel stream $\left(\mathrm{kg} / \mathrm{m}^{3}\right)$ and $\rho_{\mathrm{gv}}$ is the density of the guide vane stream $\left(\mathrm{kg} / \mathrm{m}^{3}\right)$. The MFR has been found to be the controlling parameter for mixing and resultant combustion in our previous studies [6,7]. Syngas used in this study has a composition of $\mathrm{CO}-20 \%, \mathrm{H}_{2}-20 \%, \mathrm{CO}_{2}-12 \%, \mathrm{CH}_{4}-2 \%$ and the rest $\mathrm{N}_{2}$, with a $\mathrm{LHV}$ of $\sim 5 \mathrm{MJ} / \mathrm{kg}$. The baseline temperature distributions at the exit for different MFRs are shown in Fig. 12. It can be seen that the temperature distribution is highly non-uniform due to the incomplete mixing in the mainstream. The pattern factor values shown in the legend are very high $\sim 1.5$ due to this non-uniformity. It is also worthwhile to note that the pattern factor does not change considerably with MFR as the peak temperature drops considerably as well. Based on the pattern factor values reported above, it is obvious that there is a need to investigate methods to enhance the combustor performance. The high pattern factor values are direct consequences of poor mixing and lack of combustion in the mainstream. We have explored the strategy of premixing in the mainstream to study the possibility of combustion in the mainstream flow. It must be noted here that the performance enhancement is sought based on the exhaust pattern factor. A low value of pattern factor is desirable, denoting low difference in the maximum and average temperatures. Typical desirable values for gas turbine combustors are $\leq 0.1$. The emission measurements are reported in a subsequent section for all configurations. Agarwal and Ravikrishna [5] carried out studies on combustor performance enhancement with methane fuel by employing struts in the mainstream flow. The struts consist of a plate with 6 slits with blockage area of $30 \%$. The flow past the struts results in high turbulence and generation of trailing vortices. As a result of this, the mixing of the cavity combustion products with the mainstream is significantly enhanced. The effect of struts in performance enhancement with syngas operation is studied in the current work. Figure 11(b) shows the configuration of the struts used. The struts are placed at an angle of $45^{\circ}$ to the bottom face of the combustor. Table 2 shows the conditions for which the performance enhancement has been evaluated.

The left panel in Fig. 13 shows the temperature distribution with mainstream premixing for cases 4 and 5 in Table 2. It can be seen that there is a negligible effect of mainstream fuel addition on the exit pattern factor. GTP-16-1015 Sesha Giri 
This denotes that the fuel addition in the mainstream does not undergo complete combustion and as a result the pattern factor does not improve. However, the unburnt $\mathrm{CO}$ in the fuel causes the pollutant emissions to increase considerably which is shown in Table 2 .

The right panel in Figure 13 shows the temperature distribution without and with struts. It is clear that the introduction of struts in the mainstream tends to improve the pattern factor. The pattern factor values drop from 1.4 to 0.2 in the case of MFR=4.5, in the presence of struts. This is seen in the case of MFR=1.1 as well. The recirculation zone in the downstream of the struts stabilizes the combustion in the mainstream flow. The mainstream equivalence ratio is $\sim 0.07$ in the case of $\mathrm{MFR}=4.5$. In the absence of struts, the flame is not stabilized in the mainstream. However in the presence of the struts, the recirculation zone provides a flame stabilization location in the mainstream. This is evident from Fig. 14 where the broadband flame luminosity images for $\mathrm{MFR}=4.5$ for different configurations are shown. Table 2 summarizes the typical exhaust measurements with premixing and struts. The combustion efficiency is calculated based on the exhaust $\mathrm{CO}$ and THC emission levels. For MFR=4.5 with premixing and with struts, the $\mathrm{CO}$ emissions are higher than the measurement range of the analyser. It must be noted that $\mathrm{NO}$ emissions are very low $(<3 \mathrm{ppm})$ for all cases. High THC levels in Table 1 denote incomplete combustion of the fuel in the combustor. The THC emissions are pretty much constant over different MFRs without premixing and struts. However, when the premixing is introduced in the mainstream, considerable portion of the fuel leaves the combustor unburned as evidenced by the high CO and THC levels. This situation is not remedied even by the introduction of the struts, which in spite of improving the pattern factor, does not ensure complete combustion of the excess fuel added in the mainstream. This is consistent with observations made by Agarwal and Ravikrishna [5], that struts improve the mixing of the mainstream and the products from the cavity which is reflected on the pattern factor improvement. In addition, the increase in THC levels is confirmed by the $12 \mathrm{ppm}$ THC levels in the case of MFR 4.5 with struts and no premixing (not shown here). The combustion efficiencies are also very similar for the different MFRs without premixing and struts. In the cases with premixing, the excess fuel is not burned due to the short combustion chamber length and high $\mathrm{CO}$ levels can be seen to support this. The mainstream premixing and struts configuration is most favorable for operation of the combustor in terms of pattern factor enhancement. However, it is essential to ensure lower emissions by providing a higher residence time by increasing the combustor length to completely burn the extra fuel.

The previous section detailed the exhaust measurement of temperature and pollutant emissions at the exit section of the trapped vortex combustor and enhancement was sought using mixing enhancement struts and premixing of fuel in the mainstream flow. This led to lower pattern factors in addition to ultra-low NOx and reasonably low CO and THC emissions. Numerical simulations on the TVC are conducted to explain the experimentally observed trends and the same is explained in the next few sections.

\section{Insights on exhaust measurements from simulations}

This section focuses on a comparison of the exhaust measurements with results from LES simulations. It must be noted that the numerical simulations were carried out for the baseline case mentioned in Table 1 without struts or premixing. The top-left panel in Fig 15 shows the comparison of the temperature distribution at the GTP-16-1015 
exit MFRs of 4.5 and 1.1. It can be seen that the exhaust temperatures are predicted with considerable accuracy $(\sim 150 \mathrm{~K})$. The exhaust pattern factor values are different in the case of simulations $(\sim 1.8)$, however the value remains constant across MFRs which captures the trend observed in the measurements.

The most important point to note here is that the distribution is predicted with considerable accuracy with the trend captured precisely. The offset in the peak temperature value can be attributed to the chemical kinetic mechanism as observed in simulations on chnA validation flame [9]. Considering Fig. 7, it can be seen that the $\mathrm{OH}$ concentration at the exit is higher indicating a higher temperature. However the peak value is restricted to a very thin region close to the bottom wall of the combustor. This is reflected in the temperature distribution and a steep gradient is observed in the case of $\mathrm{MFR}=4.5$. In the case of MFR=1.1, Fig. 8, shows a lower $\mathrm{OH}$ concentration value at the exit section distributed over a broader region along the height of the combustor exit. This again reflects in a flatter gradient of temperature. The reason for poor pattern factor can be attributed to the very thin reaction zone in the cavity as evidenced by the $\mathrm{OH}$ distribution in Fig. 7. As the MFR is reduced and the flame transitions to a partially premixed flame front, a distributed flame front is observed leading to a broader temperature distribution at the exit as seen in the case of MFR=1.1. The top-right panel in Fig. 15 shows the comparison of mean $\mathrm{CO}$ emission at the exit between simulations and measurements. Measurements show that with increasing MFRs the CO emissions increase and reach a peak of $\sim 1600 \mathrm{ppm}$. This is due to the non-premixed nature of the flame at high MFRs leading to the CO in the fuel to be left unburnt. On the contrary, at low MFRs, as the flame becomes progressively premixed, the CO levels drop to $\sim 200 \mathrm{ppm}$. This has been outlined in the previous section. The bottom panel shows THC emission levels for different MFRs. The THC emissions do not vary much with MFR as the hydrocarbon content in the fuel is low $\sim 2 \%$. Owing to the higher reactivity as compared to $\mathrm{CO}$, the hydrocarbon emission levels do not depend on the flame configuration and show a near-constant value. It must be noted that the $\mathrm{CO}$ and THC values reported here are both spatially and temporally averaged. In summary, the simulations provide an insight into the reason for the trends observed in exhaust measurements, attributable to the mode of flame stabilization.

\section{Conclusions}

The present work reports performance of a model trapped vortex combustor fuelled by low calorific value syngas fuel in terms of exhaust temperature distribution (pattern factor) and pollutant emissions. Numerical simulations of syngas combustion in a trapped vortex combustor are also carried out using both RANS and LES approaches incorporating the presumed PDF model to account for the turbulence-chemistry interaction. In general, LES simulations tend to agree reasonably well with measurements. This is attributed to the better prediction of flow field by LES as evidenced by the velocity magnitude contours which match well with PIV velocity measurements. With respect to comparison of exhaust measurements, the simulations indicate that temperature distribution, $\mathrm{CO}$ and THC variation with MFRs can be effectively explained based on the flame stabilization mechanisms. The NO emissions are found to be constantly below 3-ppm primarily across various flow conditions due to the low temperatures in the combustor. It is observed that mixing enhancement using struts (30\% blockage area) considerably lowers the pattern factor $(\sim 0.2)$. It is found that the mainstream 
premixing does not alter the pattern factor significantly; however the $\mathrm{CO}$ and $\mathrm{THC}$ emissions tend to increase. This is attributed to the flame stabilization in the low velocity wake regions behind the struts, which is confirmed by broadband flame luminosity images. Future efforts can focus on further enhancement of the mixing in the mainstream to obtain complete combustion.

\section{References}

[1] Singhal, A., Ravikrishna, R. V., 2011, "Single Cavity Trapped Vortex Combustion Dynamics: Part-1: Experiments," International Journal of Spray and Combustion Dynamics, 3(1), pp. 23-44

[2] Singhal, A., Ravikrishna, R. V., 2011, "Single Cavity Trapped Vortex Combustion Dynamics: Part-2: Simulations," International Journal of Spray and Combustion Dynamics, 3(1), pp. 45-52

[3] Agarwal, K. K., and Ravikrishna, R. V., 2011, "Experimental and Numerical studies in a Compact Trapped Vortex Combustor: Stability Assessment and Augmentation," Combustion Science \& Technology, 183(12), pp. $1308-1327$

[4] Agarwal, K. K., and Ravikrishna, R. V., "Validation of a Modified Eddy Dissipation Concept Model for Stationary and Non-Stationary Diffusion flames," Combustion Science and Technology, 151-164, 184(2) (2012)

[5] Agarwal, K. K., and Ravikrishna, R. V., 2013, "Mixing Enhancement in a Compact Trapped Vortex Combustor, Combustion Science and Technology," 185(3), pp. 363-378

[6] Krishna, S. and Ravikrishna, R. V., 2015, "Optical Diagnostics of Fuel-air mixing and vortex formation in a cavity combustor," Experimental Thermal and Fluid Science, 61, pp. 163-176

[7] Krishna, S. and Ravikrishna, R. V., 2015, "Quantitative OH PLIF diagnostics of syngas and methane combustion in a cavity combustor," Combustion Science and Technology, 187(11), pp.1661- 1682

[8] Li, J., Zhao, Z., Kazakov, A., Chaos, M., Dryer, F. L., Scire, J. J. Jr,. 2009, “A comprehensive kinetic mechanism for $\mathrm{CO}, \mathrm{CH}_{2} \mathrm{O}$, and $\mathrm{CH}_{3} \mathrm{OH}$ Combustion," International Journal of Chemical Kinetics, 39(3), pp. $109-136$

[9] Cuoci, A., Frassoldati, A., Ferraris, G. B., Faravelli, T., Ranzi, E., 2007, "The ignition, combustion and flame structure of carbon monoxide/hydrogen mixtures. Note2: Fluid Dynamics and kinetic aspects of syngas combustion,” International Journal of Hydrogen Energy 32, pp. 3486-3500

[10] Barlow, R. S., Fiechtner, G. J., Carter, C. D., Chen, J.-Y., 2000, "Experiments of the scalar structure of turbulent $\mathrm{CO} / \mathrm{H}_{2} / \mathrm{N}_{2}$ Jet flames," Combustion and Flame 120, pp. 549-569

[11] Kemenov, K. A., Wang, H., and Pope, S. B., 2009, “Grid resolution effect on LES of piloted methane-air flame," Proceedings of 6th US National Combustion Meeting, pp. 146-159

[12] Boudier, G., Gicquel, L. Y. M., Poinsot, T., Bissieres,. D and Berat, C., 2009, "Effect of mesh resolution on large eddy simulation of reacting flows in complex geometry combustors," Combustion and Flame, 155(12), pp. 196-214,146-159 
[13] Jin, Y., Li, Y., Xiaomin He, Zhang, J., Jiang, B., Wu, Z., Song, Y., 2014, “Experimental investigations on flow field and combustion characteristics of a model trapped vortex combustor," Applied Energy, 134, pp. 257-269

[14] Medhat, A., Nemitallah, Mohamed A. H., 2013, "Experimental and numerical investigations of an atmospheric diffusion oxy-combustion flame in a gas turbine model combustor," Applied Energy, 111, pp $401-415$

[15] Zeinivand, H., Bazdidi-Tehrani, F., 2012, "Influence of stabilizer jets on combustion characteristics and NOx emission in a jet-stabilized combustor"Applied Energy, 92, pp. 348-360

[16] Zhang, R.C., Fan, W. J., Shi, Q., Tan, W. L., 2014, “Combustion and emissions characteristics of dualchannel double-vortex combustion for gas turbine engines," Applied Energy, 130, pp. 314-325

[17] Arghode, V. K., Gupta, A. K., 2013, "Role of thermal intensity on operational characteristics of ultra-low emission colourless distributed combustion,” Applied Energy, 111, pp. 930-956

[18] Krishna, S., Santanu Pramanik and Ravikrishna, R. V., 2013, "Numerical modelling of a Turbulent NonPremixed CO/H2/N2 flame," 23rd National Conference on IC Engines and Combustion, 


\begin{tabular}{|c|c|c|c|c|c|}
\hline \multicolumn{7}{|c|}{ Simulation conditions } \\
\hline S. No & $V_{\text {cf }}(\mathrm{m} / \mathrm{s})$ & $\mathrm{V}_{\mathrm{gv}}(\mathrm{m} / \mathrm{s})$ & MFR & $\varphi_{\mathrm{c}}$ & $\varphi_{\text {overall }}$ \\
\hline $\mathbf{1}$ & 6.11 & 2.85 & 4.45 & 0.96 & 0.16 \\
\hline $\mathbf{2}$ & 5.61 & 5.20 & 1.13 & 0.48 & 0.1 \\
\hline $\mathbf{3}$ & 4.08 & 7.54 & 0.3 & 0.24 & 0.05 \\
\hline
\end{tabular}

Table 1: Non-reacting and reacting flow conditions for simulations

\begin{tabular}{|c|c|c|c|c|c|c|}
\hline Case no. & MFR & $\mathbf{C O}(\boldsymbol{\%})$ & $\begin{array}{c}\mathbf{C O}_{2} \\
(\boldsymbol{\%})\end{array}$ & $\begin{array}{c}\text { THC } \\
(\mathbf{p p m})\end{array}$ & $\begin{array}{c}\text { NO } \\
(\mathbf{p p m})\end{array}$ & $\begin{array}{c}\boldsymbol{\eta} \text { comb } \\
(\%)\end{array}$ \\
\hline 1 & 4.5 & 0.16 & 6.5 & 14 & 3 & 96.2 \\
\hline 2 & 1 & 0.05 & 3.3 & 15 & 1 & 97.5 \\
\hline 3 & 0.3 & 0.03 & 2.2 & 14 & 1 & 98 \\
\hline 4 & $4.5-$ premixing & $>0.2$ & 3 & 300 & 1 & - \\
\hline 6 & $4.5-$ premixing and & $>0.2$ & 6 & 354 & 1 & - \\
\hline
\end{tabular}

Table 2: Exhaust performance characteristics for different conditions 
List of Figures
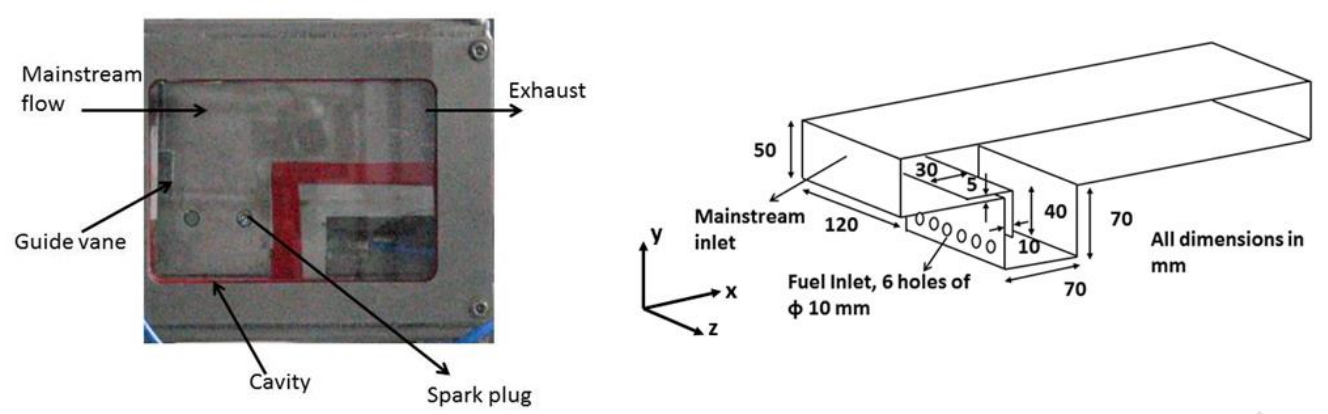

Figure 1: Single cavity TVC combustor rig and schematic with dimensions
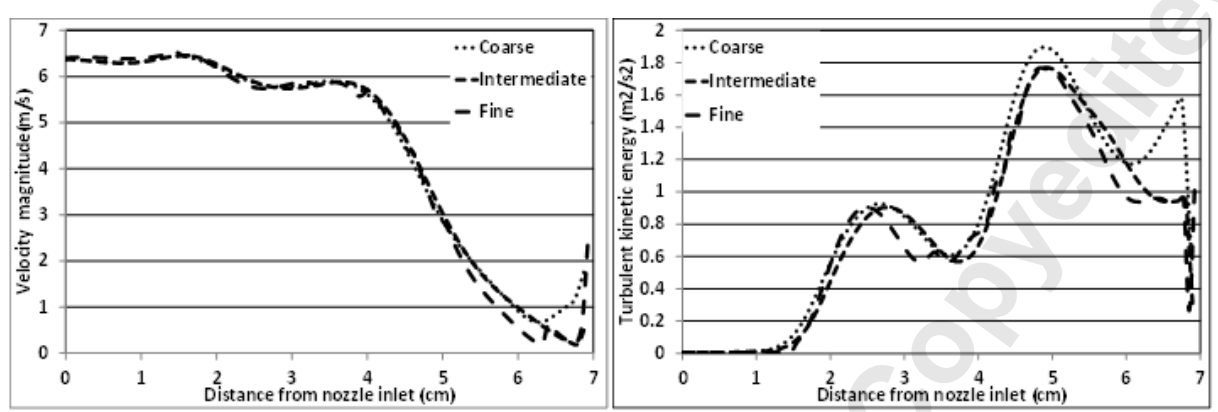

CCM-RANS

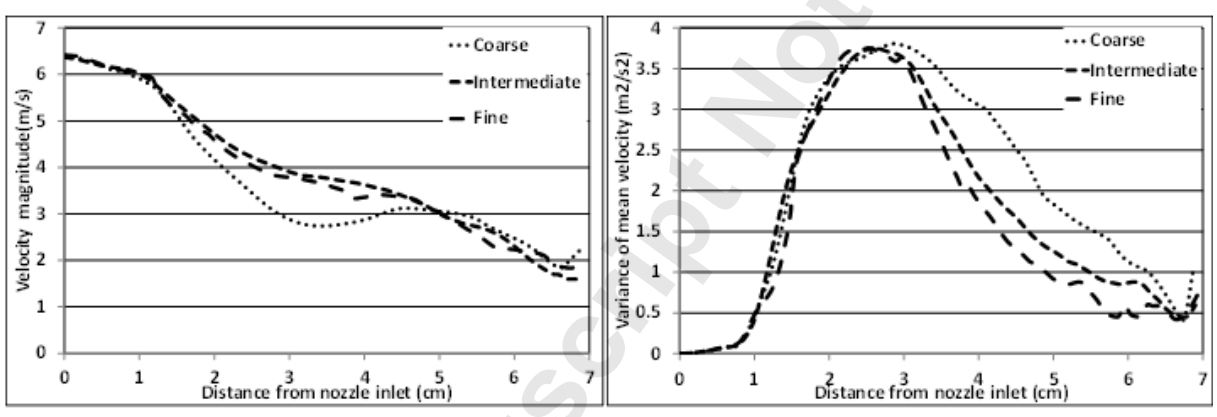

CCM-LES

Figure 2: Grid independence results for RANS and LES simulations

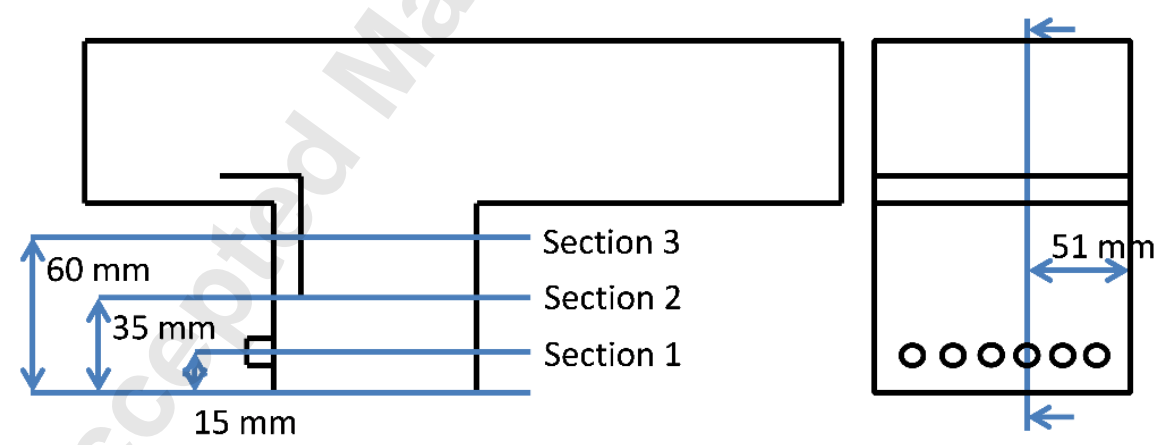

Figure 3: Stations for comparison of simulations and experimental results 


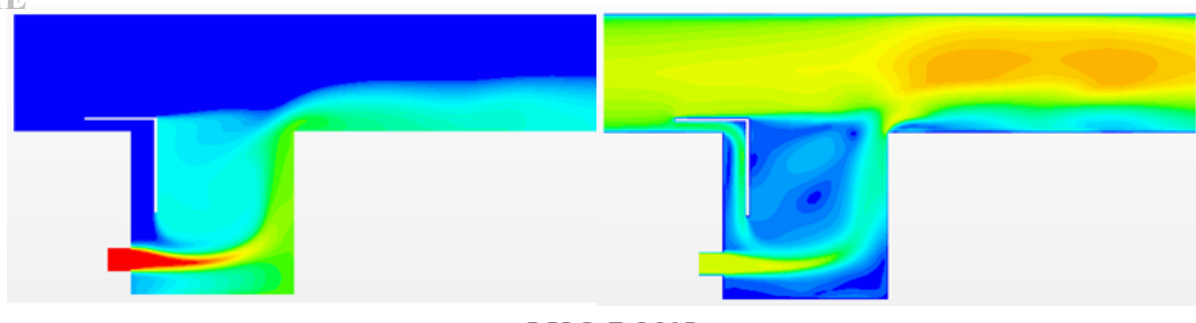

CCM-RANS

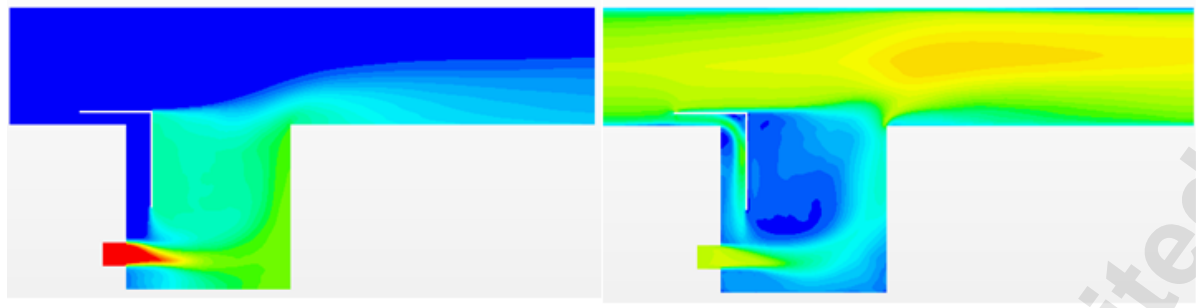

\section{CCM-LES}

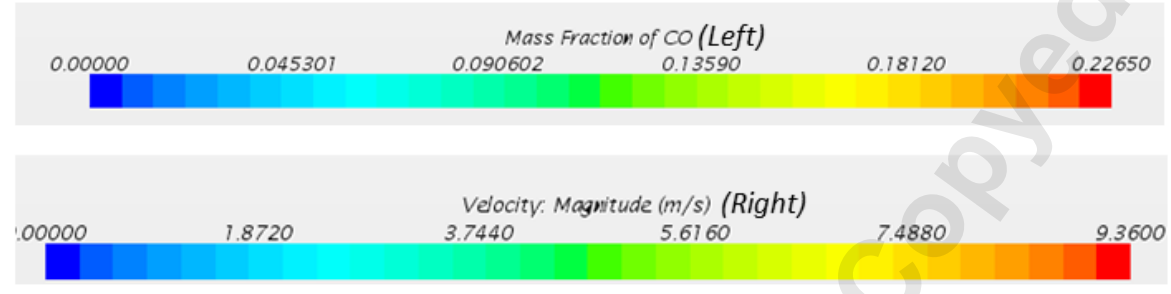

Figure 4: CO mass fraction and velocity magnitude contours for Case 1 (MFR 4.5) 


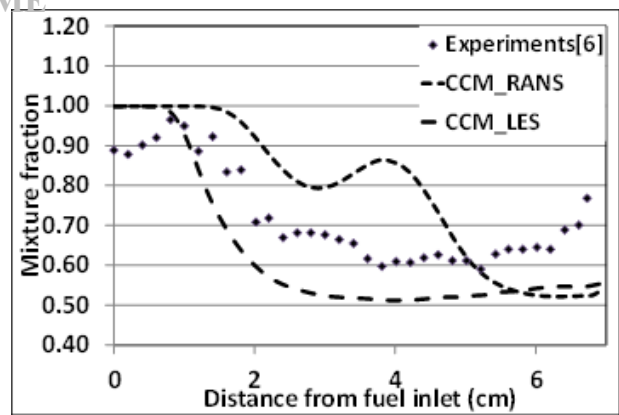

Section 1 : Fuel inlet

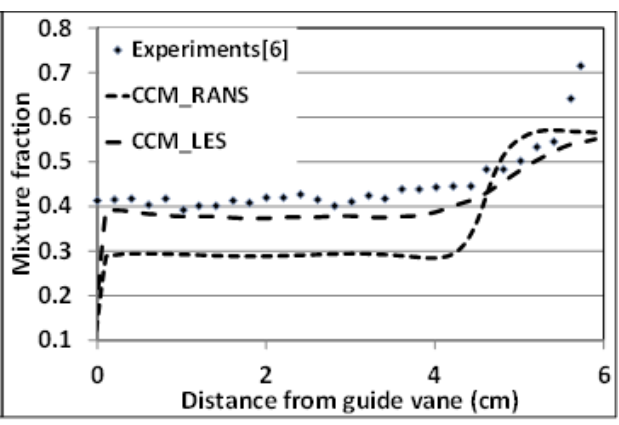

Section 2: Cavity centre

Case 1: MFR 4.5

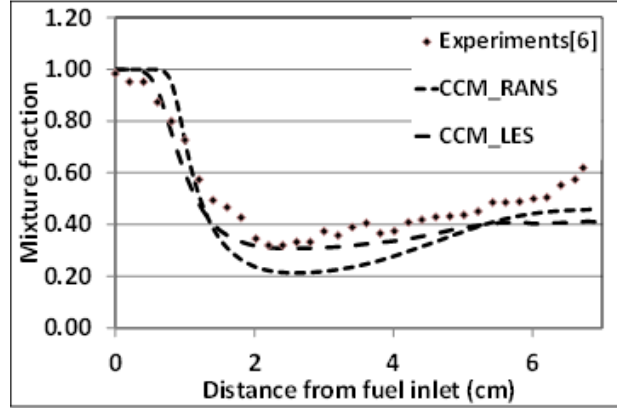

Section 1 : Fuel inlet

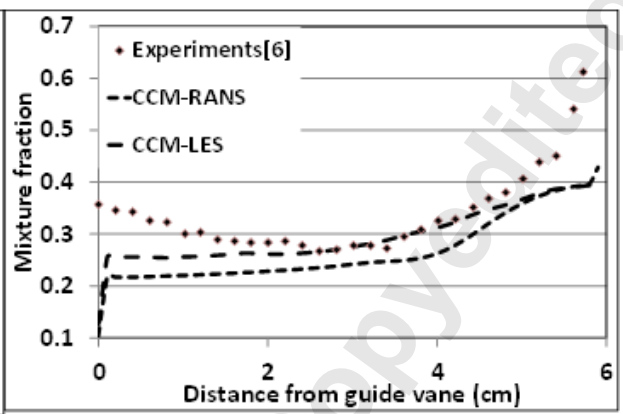

Section 3: Cavity exit

Case 2: MFR 1.1

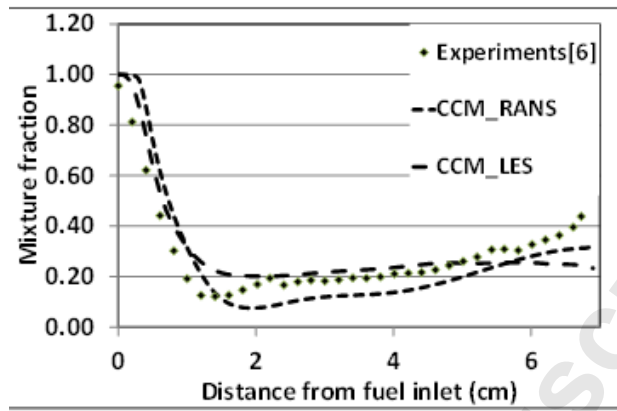

Section 1 : Fuel inlet

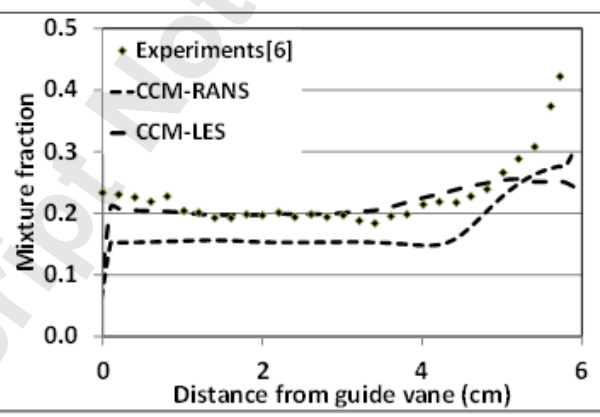

Section 2: Cavity center

Case 3: MFR 0.3

Figure 5: Mixture fraction profiles at different sections for MFR 4.5, 1.1 and 0.3 


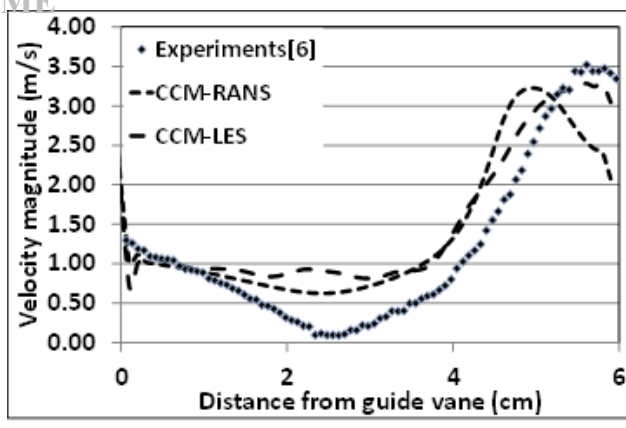

Case 1: MFR 4.5

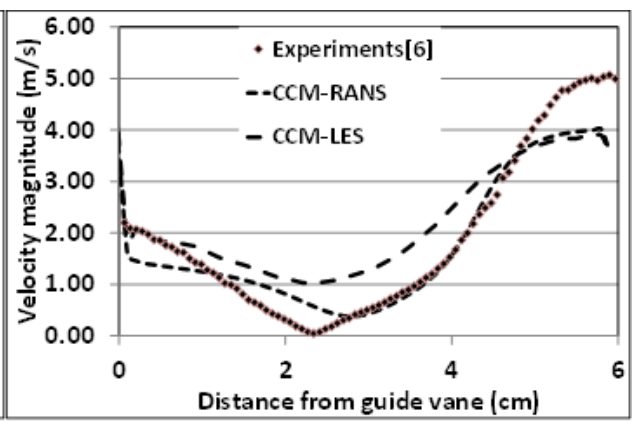

Case 2: MFR 1.1

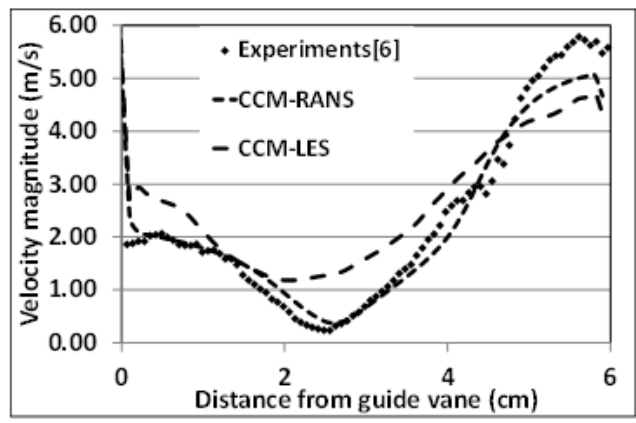

Case 3: MFR 0.3

Figure 6: Velocity magnitude profiles at Section 2 (cavity centre) for MFR 4.5, 1.1 and 0.3
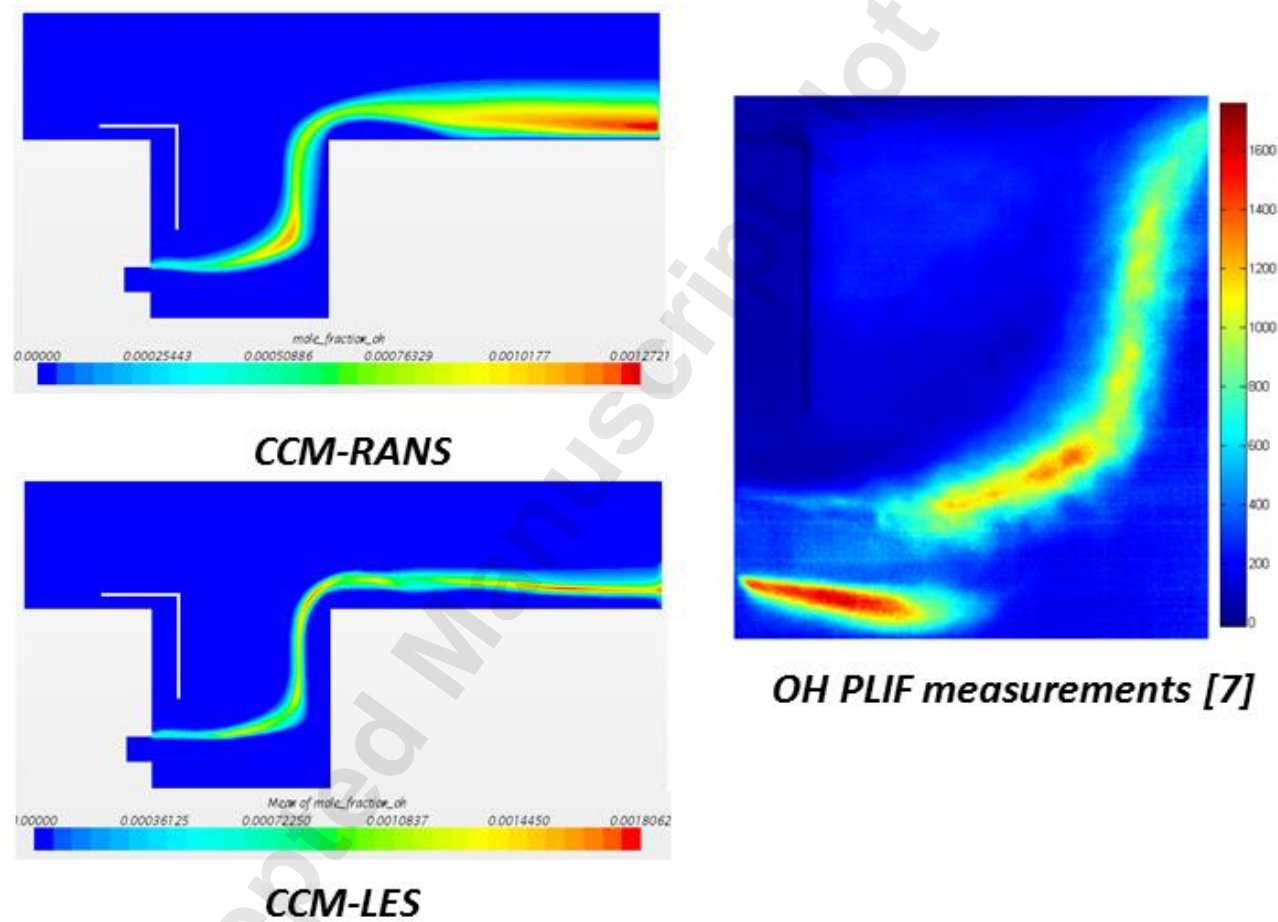

OH PLIF measurements [7]

Figure 7: $\mathrm{OH}$ concentration contours for Case 1 (MFR 4.5) 


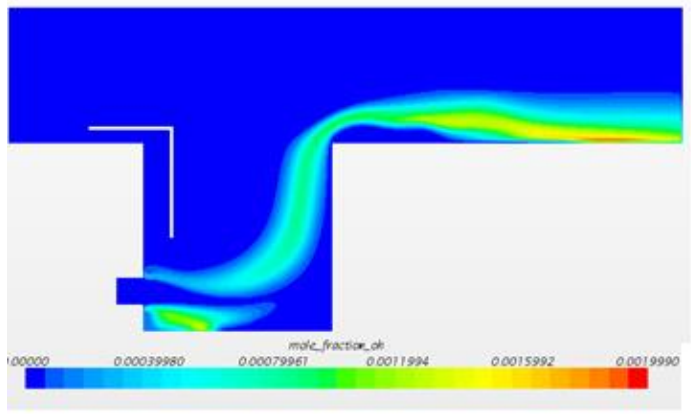

CCM-RANS
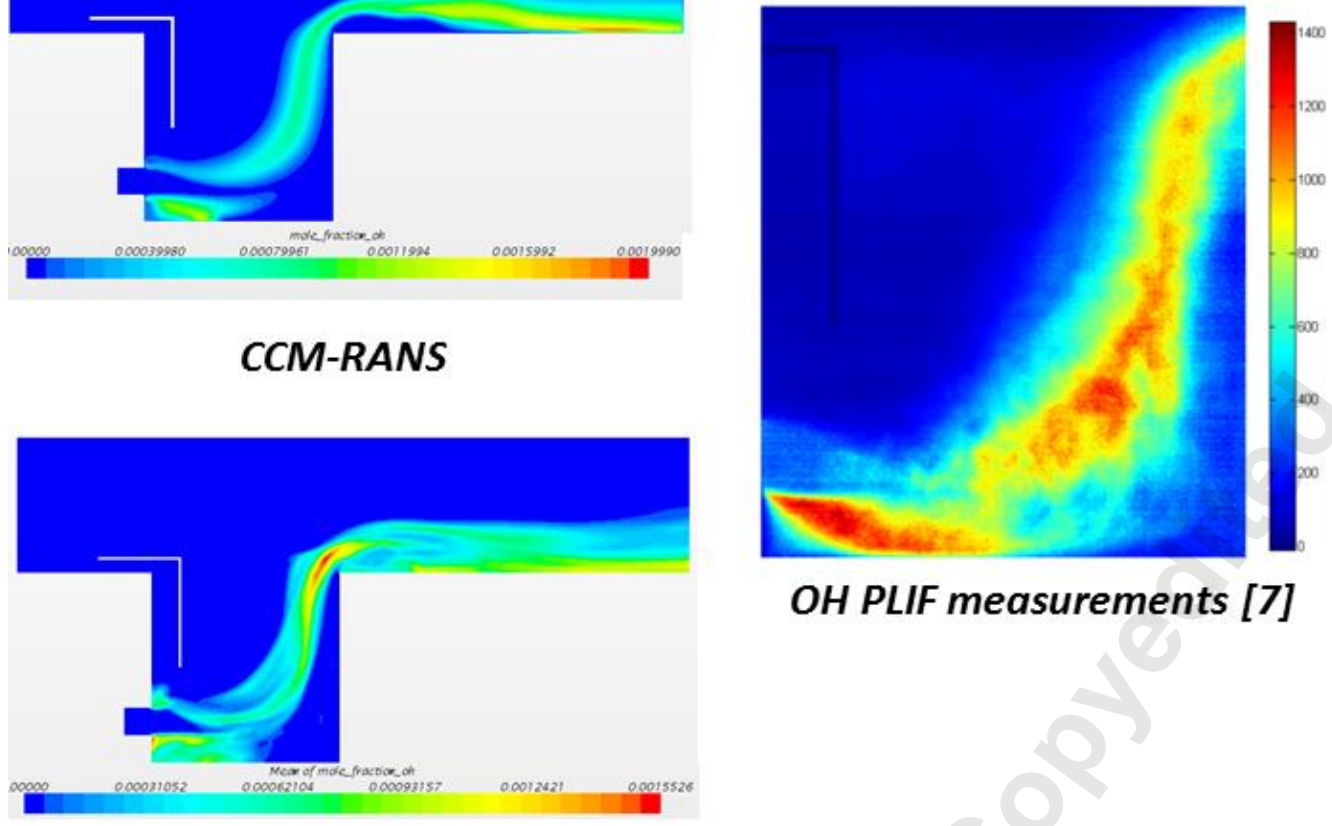

OH PLIF measurements [7]

\section{CCM-LES}

Figure 8: OH concentration contours for Case 2 (MFR 1.1) 


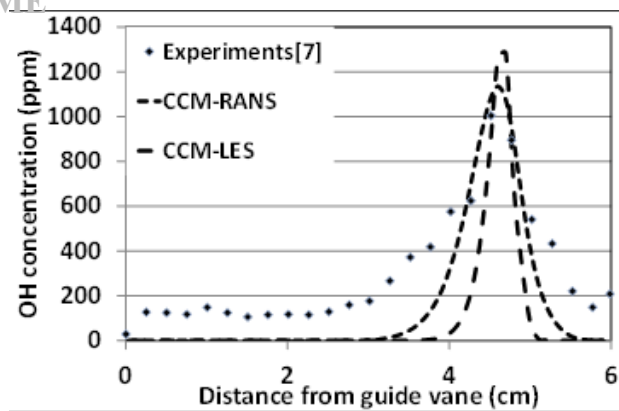

Section 2 : Cavity Centre

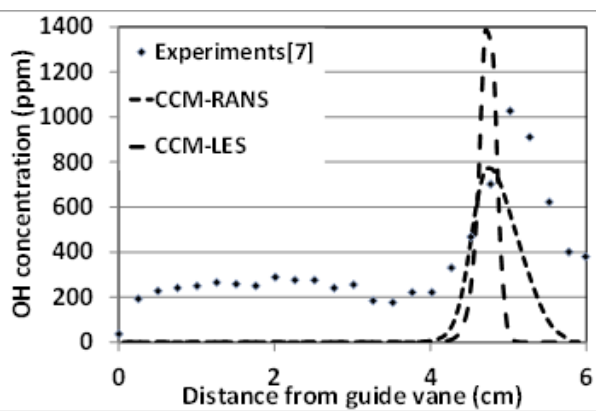

Section 3 : Cavity exit

MFR 4.5

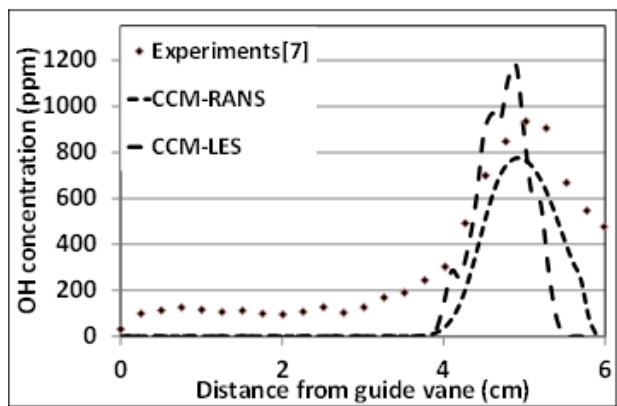

Section 2 : Cavity Centre

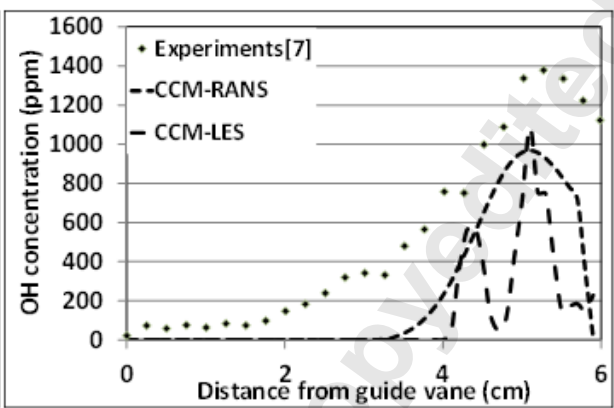

Section 3 : Cavity exit

MFR 1.1

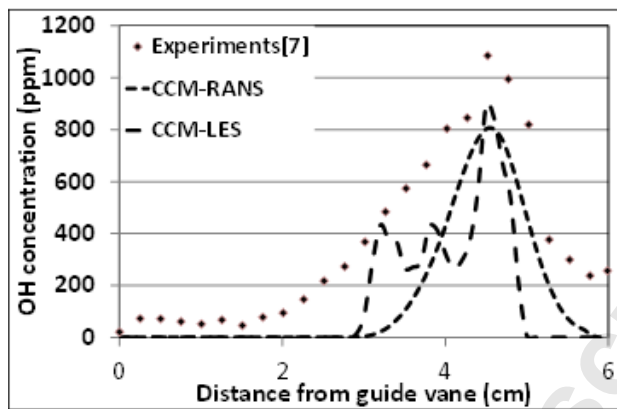

Section 2 : Cavity Centre

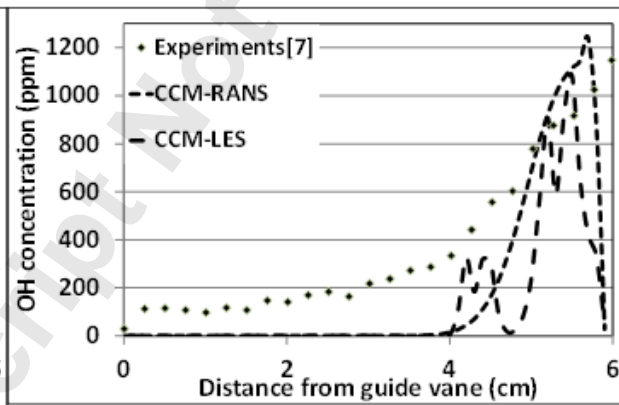

Section 3 : Cavity exit

\section{MFR 0.3}

Figure 9: $\mathrm{OH}$ concentration profiles at different sections 2 and 3 for MFR 4.5, 1.1 and 0.3 


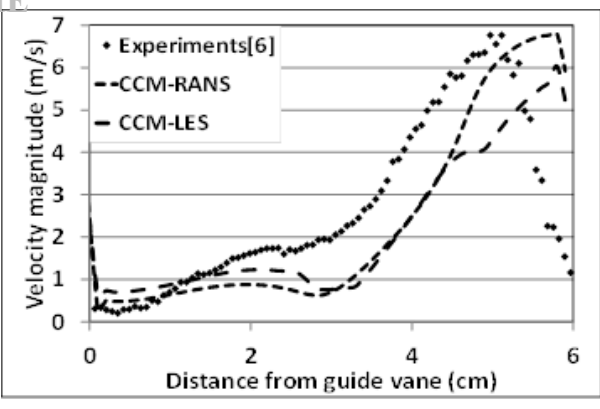

Case 1: MFR 4.5

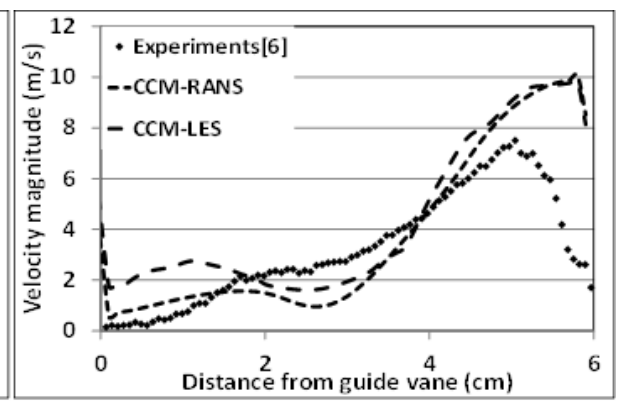

Case 2: MFR 1.1

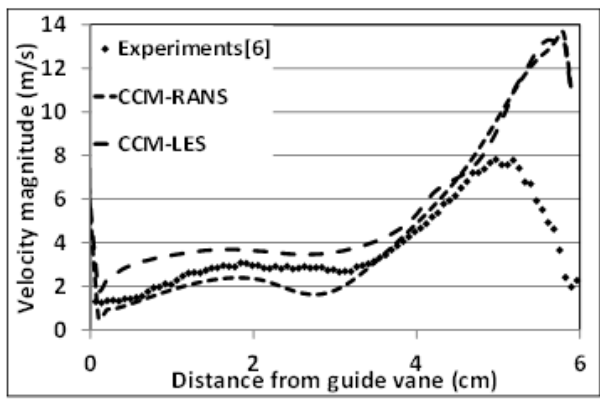

Case 3: MFR 0.3

Figure 10: Velocity magnitude profiles at Section 3 for MFR 4.5, 1.1 and 0.3

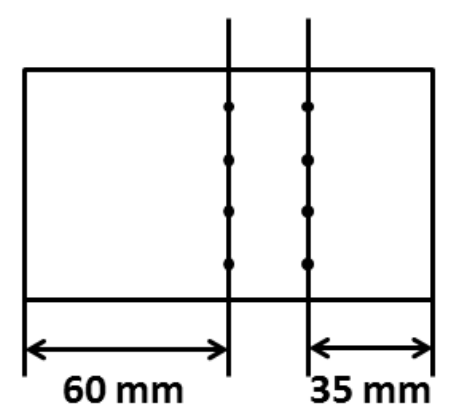

Thermocouple temperature measurement locations

(a)

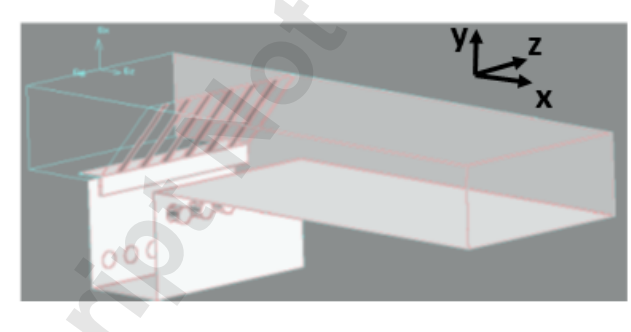

Struts in mainstream flow at $45^{\circ}$ for mixing enhancement

(b)

Figure 11: Thermocouple measurement locations and flow enhancement struts

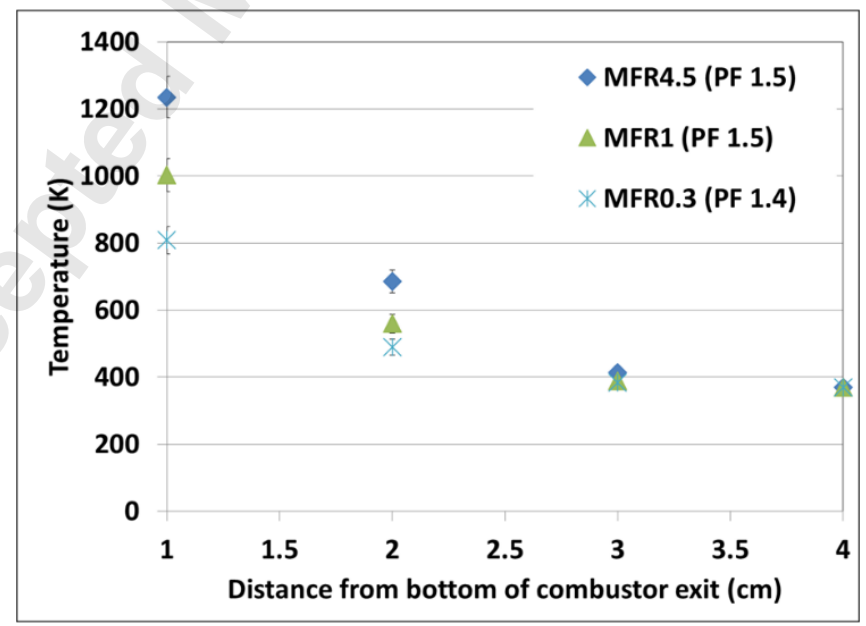

Figure 12: Baseline temperature distribution 
Journal of Engineering for Gas Turbines and Power. Received January 14, 2016;

Accepted manuscript posted June 5, 2017. doi:10.1115/1.4036945

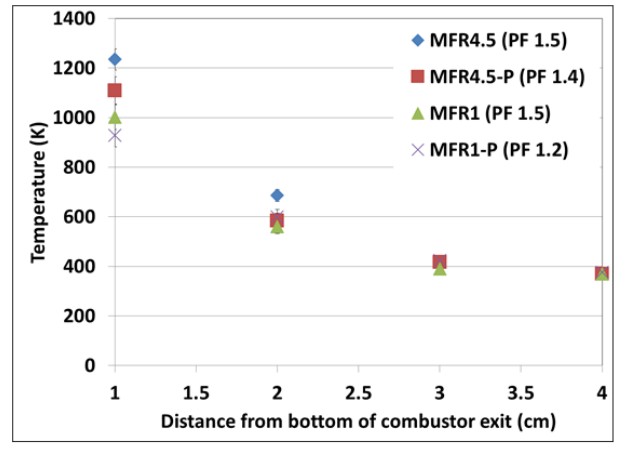

Temperature distribution with mainstream premixing

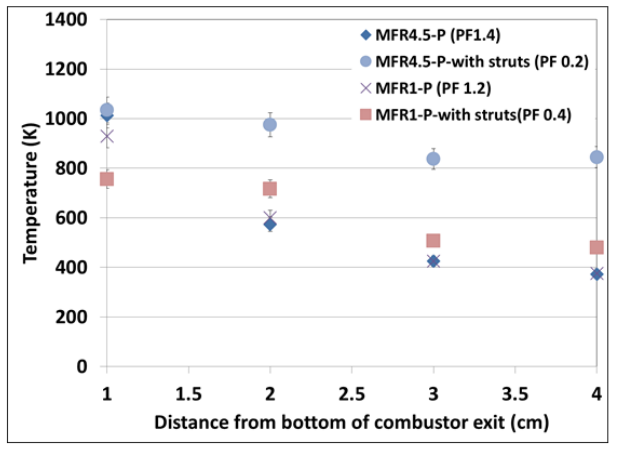

Temperature distribution with mainstream premixing and struts

Figure 13: Temperature distribution with mainstream premixing and struts

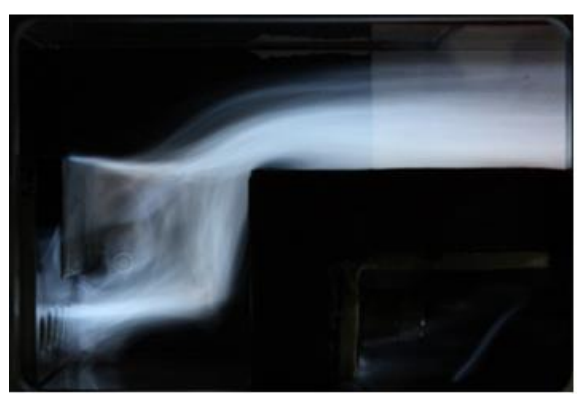

Baseline case MFR 4.5

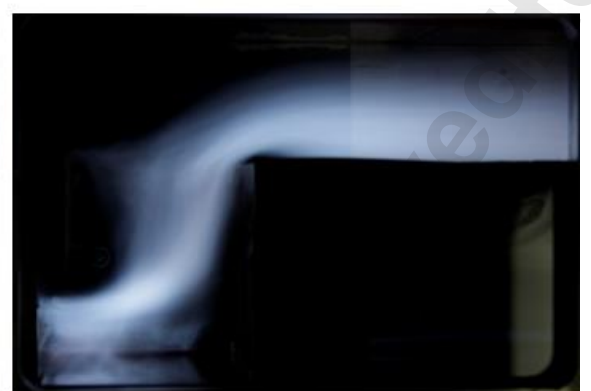

MFR 4.5 with premixing

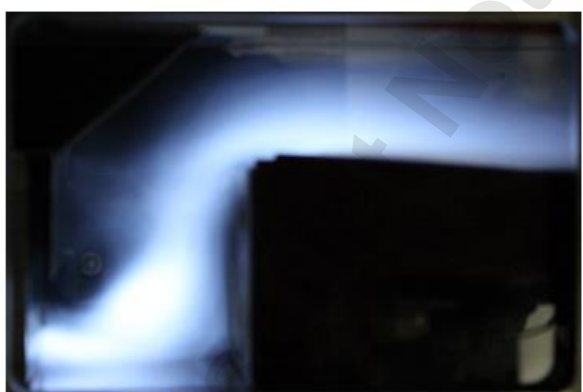

MFR 4.5 with premixing and struts

Figure 14: Broadband luminosity images for MFR 4.5 for baseline, premixing and struts cases 


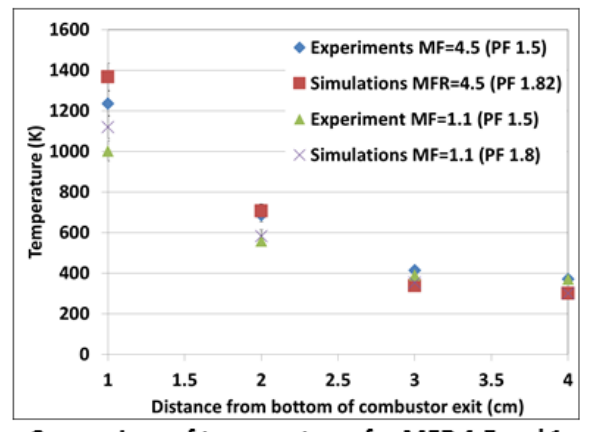

Comparison of temperatures for MFR 4.5 and 1

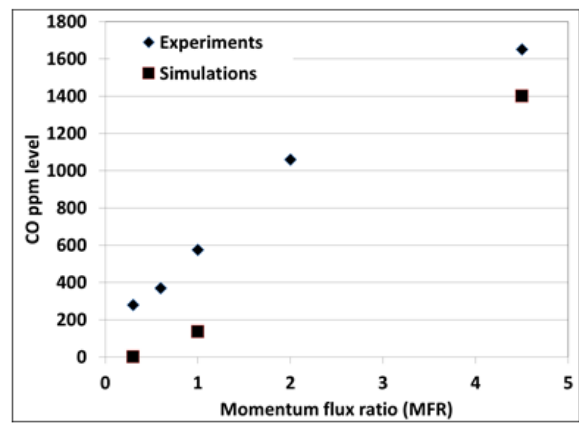

Comparison of CO levels between experiments \& simulations

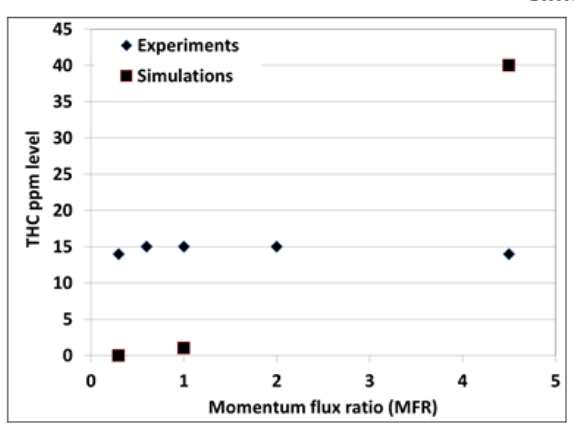

Comparison of THC levels between experiments \& simulatione

Figure 15: Comparison of temperature and pollutant emission at the exit plane for experiments and simulations 